\title{
Endoscopic resection and enucleation of gastric submucosal tumor facilitated by subsequent closure of incision using over-the-scope clip
}

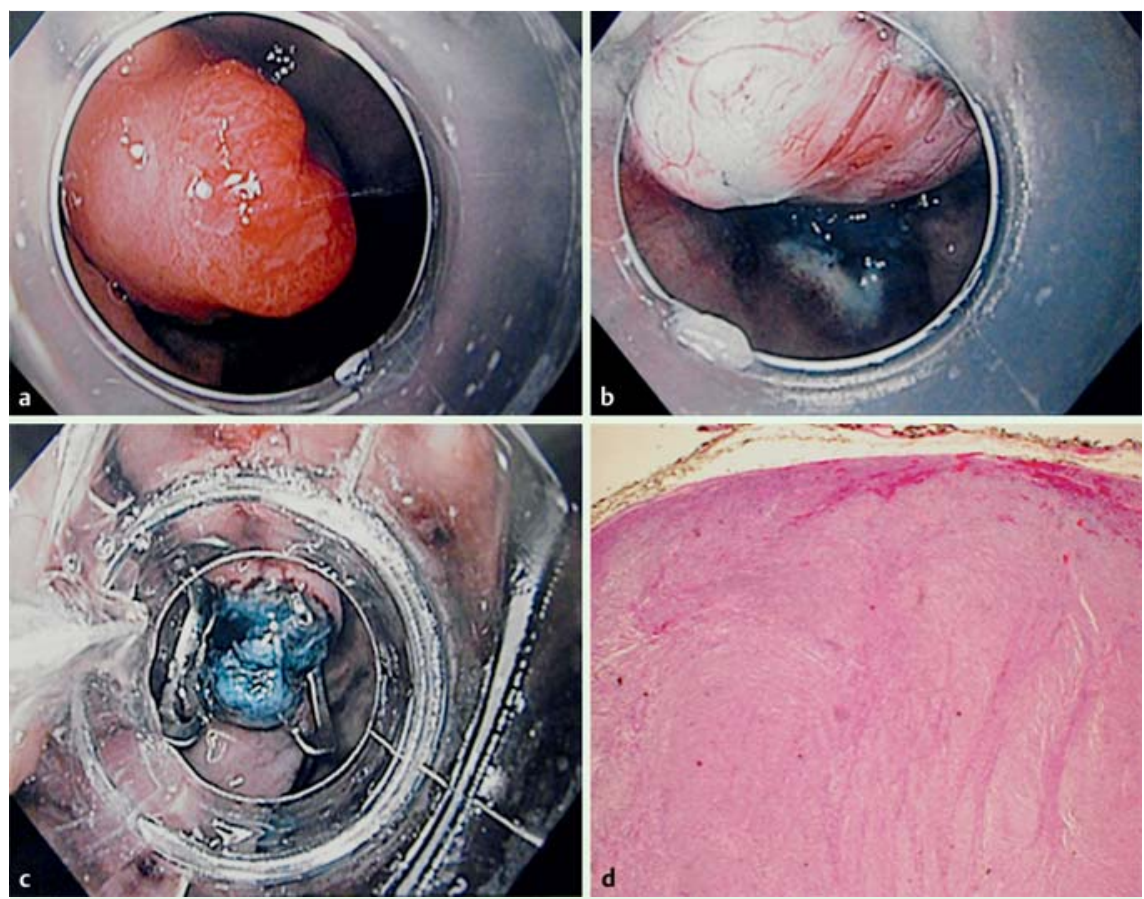

Fig. 1 Endoscopic enucleation of a gastric submucosal tumor in a 73-year-old man with a history of diabetes mellitus, hypertension, coronary artery disease, and atrial fibrillation. a A 20-mm reddish elevated lesion of the lower gastric body. $\mathbf{b}$ Careful dissection was performed, exposing the entire capsulized round tumor, which was completely excavated from its base and enucleated. $\mathbf{c}$ The over-thescope clip was released and the wound closed successfully. $\mathbf{d}$ Resected specimen of gastric leiomyoma with hyperplastic epithelium on the surface.

A 73-year-old man with a history of diabetes mellitus, hypertension, coronary artery disease, and atrial fibrillation was found to have a $20-\mathrm{mm}$ reddish elevated lesion of the lower gastric body ( Fig.1a). Endoscopic ultrasound revealed a submucosal tumor originating from the muscularis propria. The patient preferred an endoscopic excision. An endoluminal resection was performed using the endoscopic submucosal dissection (ESD) technique with carbon dioxide insufflation. An indigo carmine-saline mixture was injected into the base of the lesion. After creation of incision with the tip of a snare, the IT-knife2 (Olympus, Melville, New York, United States) was then used to circumferentially cut the mucosa and submucosa around the tumor. Careful dissection was performed, expos- ing the entire capsulized round tumor, which was completely excavated from its base and enucleated ( Fig. 1 b). The resection was deep exposing the muscle layer. Given the patient's high risk cardiovascular status and continued need for antiplatelet agents, the wound was closed endoscopically using an over-the-scope clip (OTSC; 12/6t, Ovesco, Tübingen, Germany). Air was suctioned from the stomach to decrease the surface area of the wound. Then the OTSC device was directed towards the wound and suction was applied to pull the lateral mucosa edges into the cap. The clip was released and the wound closed successfully ( $\bullet$ Fig. 1 c). The patient was discharged home and did well. The resected specimen confirmed enucleation of the gastric leiomyoma (॰ Fig. 1d).
While ESD and excavation techniques are potential therapeutic options for the removal of a gastric submucosal tumor, they more frequently result in perforation than ESD for early gastric cancer $[1,2]$. This case shows the utility of OTSC for full-wall closure during a one-step process, thus minimizing the risk of delayed bleeding and perforation. Although endoclips can be used, that is technically a more arduous and difficult procedure, usually necessitating several clips. Although the OTSC device is more expensive than a single clip, the use of more than three clips may exceed the price of an OTSC. In addition, the apposition forces of OTSC are much stronger and thus may result in a more secure closure $[3,4]$.

Endoscopy_UCTN_Code_TTT_1AO_2AG

Competing interests: None

\section{Seiichiro Abe ${ }^{1,3}$, Leona Council', Xaoiyan Cui², Yutaka Saito ${ }^{3}$, Klaus Mönkemüller ${ }^{1}$}

${ }^{1}$ Basil I. Hirschowitz Endoscopic Center of Excellence, Division of Gastroenterology and Hepatology, University of Alabama at Birmingham, Alabama, USA

2 Department of Pathology, University of Alabama at Birmingham, Alabama, USA

3 Endoscopy Division, National Cancer Center Hospital, Tokyo, Japan

\section{Acknowledgments \\ $\nabla$}

$\operatorname{Dr}$ Seiichiro Abe is the recipient of the UAB Visiting Scientist/Professor Award and performed this work during his Boston Scientific Visiting Fellowship at the Basil I. Hirschowitz Center of Endoscopic Excellence, Division of Gastroenterology, University of Alabama at Birmingham, USA.

\section{References}

1 Zhang S, Chao GQ Li M et al. Endoscopic submucosal dissection for treatment of gastric submucosal tumors originating from the muscularis propria layer. Dig Dis Sci 2013; 58: $1710-1716$

2 Li QL, Yao LQ Zhou PH et al. Submucosal tumors of the esophagogastric junction originating from the muscularis propria layer: a large study of endoscopic submucosal dis- 
section (with video). Gastrointest Endosc 2012; 75: $1153-1158$

3 Mönkemüller K, Peter S, Toshniwal J et al. Multipurpose use of the "bear claw" (overthe-scope-clip system) to treat endoluminal gastrointestinal disorders. Dig Endosc 2014; 26: $350-357$

4 Sarker S, Gutierrez JP, Council L et al. Overthe-scope clip-assisted method for resection of full-thickness submucosal lesions of the gastrointestinal tract. Endoscopy 2014; 46: $758-761$
Bibliography

DOI http://dx.doi.org/

10.1055/s-0034-1391254

Endoscopy 2015; 47: E153-E154

(C) Georg Thieme Verlag KG

Stuttgart · New York

ISSN 0013-726X

\section{Corresponding author}

Klaus Mönkemüller, MD, PhD,

Professor of Medicine and Director

Basil I. Hirschowitz Endoscopic Center of Excellence

University of Alabama at Birmingham

Endoscopy Unit

JT 664

619 19th Street S

Birmingham

AL 35249

USA

klaus1@uab.edu 